\title{
Regulatory T-cells in Acquired Aplastic Anaemia
}

\author{
Mehwesh Taj ${ }^{1}$, Saima Munzir ${ }^{2}$, Sidra Maqsood ${ }^{3}$, Sumaira Shareef ${ }^{3}$, Tasneem Farzana ${ }^{4}$ and Tahir Shamsi ${ }^{1,4}$ \\ ${ }^{1}$ Department of Clinical Haematology, National Institute of Blood Disease \& Bone Marrow Transplantation, Karachi, Pakistan.
}

${ }^{2}$ Department of Transplant Immunology, National Institute of Blood Disease \& Bone Marrow Transplantation, Karachi, Pakistan.

${ }^{3}$ Department of Clinical Research, National Institute of Blood Disease \& Bone Marrow Transplantation, Karachi, Pakistan.

${ }^{4}$ Department of Bone Marrow Transplantation, National Institute of Blood Disease \& Bone Marrow Transplantation,

Karachi, Pakistan.

\begin{abstract}
Introduction: Acquired aplastic anaemia is characterized by destruction of haematopoietic stem cells by cytotoxic T lymphocytes. Regulatory T-cells have been reported to be suppressed in aplastic anaemia like many other autoimmune disorders. Haematopoietic response and haematological recovery after successful immunosuppressive treatment provide the most powerful evidence for the immune mediated basis of this complex disorder.
\end{abstract}

Objective: The aim of this study is to determine the deficiency of CD4+ CD25+ T regulatory cells prior to ATG induction/therapy in patients of aplastic anaemia.

Methods: The patients were enrolled in a dose finding study for ATG-Fresenius (Fresnious Biotec, Gernmany). Aplastic anaemia was diagnosed as per standard guidelines. Bone marrow trephine biopsy was done to assess disease severity and for classification according to Camitta classification. Flow cytometry was done using four colour flow cytometer FACSCaliber.

Results: The median age of the patients was 23 years (range 7-63 years). Out of 18 patients, there were 12 (66\%) males and 6 (34\%) females. Of the 18 evaluable patients, the number of patients with NSAA, SAA, and VSA was 10, 5, and 3 respectively. CD4+ and CD8+ T cells, and B cells in peripheral blood were calculated. The pre-treatment average Treg frequencies in NSAA was 16.5 \pm 18.8, SAA 29.1 \pm 18.3 and VSAA was $12 \pm 11.7$ respectively. The median time from diagnosis up to the last follow-up was 1.1 years (range: 0.13 .0 ).

Conclusion: The study concluded that Tregs were decreased in all patients of acquired aplastic anaemia, as in other autoimmune diseases.

Keywords: Anti-Thymocyte globulin, Aplastic anaemia, Flow cytometry, Regulatory T-lymphocytes, T-cells.

\section{REGULATORY T-CELLS IN ACQUIRED APLASTIC ANAEMIA IN PAKISTAN POPULATION INTRODUCTION}

Acquired Aplastic Anaemia (AA) is the commonest type of life threatening bone marrow failure syndromes. It is characterized by apoptosis of CD34+ hematopoietic cells of all lineages, by autologous $\mathrm{T}$ lymphocytes (which include CD8+ cytotoxic $\mathrm{T}$ cells and CD4+ subgroup of $\mathrm{T}$ cells), leading to a hypocellular marrow and peripheral pancytopenia $[1,2]$. Response to immunosuppressive therapy (IST), with anti-thymocyte globulin (ATG) and Cyclosporin (CsA), occurs in $60-75 \%$ of patients, pointing towards the immune mediated destruction as the pathogenesis of this entity [3].

It is an idiopathic acquired disorder characterized by an abnormal expansion, and aberrant immune response of CD8+

\footnotetext{
*Address correspondence to this author at the Department of Clinical

Haematology, National Institute of Blood Disease \& Bone Marrow Transplantation, Karachi, Pakistan. E-mail: mehweshfaisal@gmail.com
}

$\mathrm{T}$ cells. These cells secrete inflammatory cytokines, Interferon gamma (INF-Y) and Tumor necrotic factor alpha (TNF- $\alpha$ ), inducing apoptosis of CD34+ cells through Fas-dependent pathway [4]. Recent advances are suggesting the causative role of CD4+ T-cell mediated autoimmunity in this disease [5]. The major types of CD4+T cells in this regard include INF- $\Upsilon$ producing CD4+ cells (Th1), IL-4 producing CD4+ cells (Th2), regulatory $\mathrm{T}$ cells (Treg), and IL-17 producing CD4+ cells (Th17). Emerging data is demonstrative of reduced number of Treg in most of the studies $[6,7]$. The population and function of Treg vary in different autoimmune diseases [8], MDS [9] and AA [10, 11]. The qualitative and quantitative defects of Treg have been attributed to play a major role in the pathogenesis of AA in a number of studies $[6,7]$.

In the current study, quantitative analysis of pretreatment $\mathrm{T}$ cell subsets including Treg has been performed in Pakistani cohort of patients suffering from acquired AA. 


\section{METHODS}

Pre-treatment peripheral blood samples were analyzed for lymphocyte subset analysis on FACSCalibur flowcytometer (Becton \& Dickinson, USA) from patients diagnosed as acquired AA. Disease severity was established according to Camitta's criteria [12]. Pretreatment $T$ cell subset analysis was performed in all patients who received immunosuppressive therapy as part of investigator initiated trial using rabbit ATG-Fresenius (www.clinicaltrial.gov Clinical trial; NCT02028416). Patients with evidence of chromosomal breaks for Fanconi's anaemia, paroxysmal nocturnal haemoglobinuria and abnormal bone marrow cytogenetics were not included in the study. Institutional review board of National Institute of blood disease center had approved the study and samples were obtained after a signed consent of patients.

Peripheral blood sample was collected in K2-EDTA tube from each patient. Complete blood counts for all the samples were analyzed on XN1000. For T-cell subsetsCD3 / CD8 / CD45 / CD4 / TruC antibodies BD Biosciences, and for B-cell subsets CD3/ CD16+56/ CD45/ CD19 MLT/ TruC antibodies BD Biosciences were utilized according to manufacturer's protocol. These pre-conjugated monoclonal antibodies were incubated with peripheral blood samples followed by addition of cell lysing solution in the tubes. Tubes, then incubated again for 10 minutes at room temperature, were acquired on FACSCalibur by multiset software for T-cell \& B-cell subset enumeration.

For enumeration of regulatory T-cells, blood samples were treated with BD FACS lysing solution for appropriate time at room temperature followed by PBS wash thrice. Approximately 105 cells were incubated with CD45FITC/ CD4PE / CD3 PerCP/ CD25 APC pre-conjugated monoclonal antibodies (BD Biosciences) corresponding with Isotypic control tube. After incubation, cells were washed with PBS solution twice followed by acquisition on BD FACSCalibur. Final analysis was performed employing CellQuest-Pro software. We analyzed the abnormal frequencies of Treg by gating the Lymphocyte population in peripheral blood samples of each patient diagnosed with AA. Mean and standard deviations were calculated for mean, median and ranges Using SPSS version 20.

\section{RESULTS}

The detailed characteristics of study cohort are shown in (Table 1). The median age of the patients was 23 years (range 7-63 years). Of these 18 patients, 10 patients were of NSAA, 5 patients had SAA, and 3 patients were labeled as VSAA. CD4+ and CD8+ T cells, and B cells in peripheral blood were calculated. The pre-treatment average Treg frequencies in NSAA was $16.5 \pm 18.8$, SAA $29.1 \pm 18.3$ and VSAA was 12 \pm 11.7 respectively (Fig. 1). The B cells average frequency in pre-treatment samples was $169 \pm 83$; however, CD4+/CD8+ T cells average frequency in pre-treatment samples was $1.28 \pm$ 0.33 (Fig. 2). All patients had significantly decreased frequencies of Treg in peripheral blood samples prior to immunosuppressive treatment or allogenic bone marrow transplantation.

Table 1. Baseline Characteristics of Patients with AA with Flowcytometry Samples.

\begin{tabular}{|l|c|}
\hline Characteristic & Value \\
\hline Number of patients & 18 \\
\hline Median age, y (range) & $23(7-63)$ \\
\hline Disease severity at diagnosis \\
\hline $\mathrm{N}=(\%)$ & \\
\hline SAA & $5(27.7 \%)$ \\
\hline NSAA & $10(55.5 \%)$ \\
\hline VSAA & $3(16.6 \%)$ \\
\hline Sex (n) (\%) & $12(70.8 \%)$ \\
\hline Male & $6(29.1 \%)$ \\
\hline Female & 18 \\
\hline Etiology & \\
\hline Idiopathic & \\
\hline
\end{tabular}

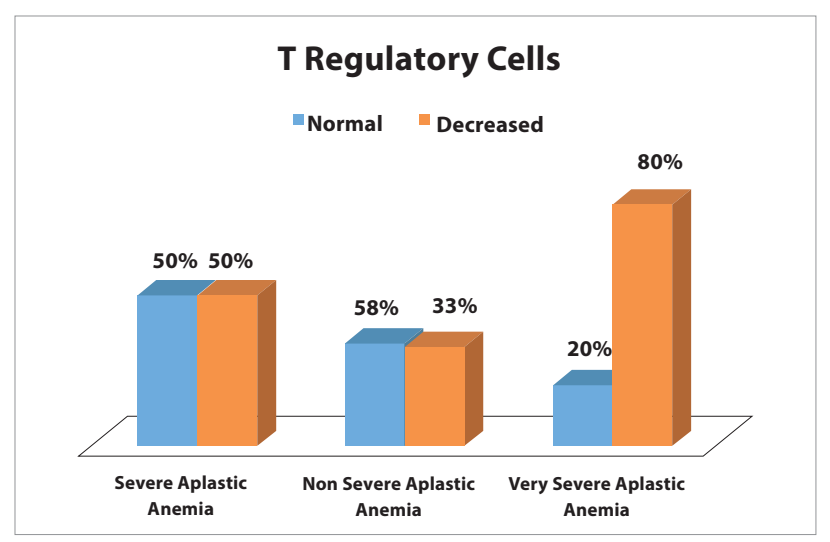

Fig. (1). Distribution of Regulatory $\mathrm{T}$ Cells in Aplastic Anemia.

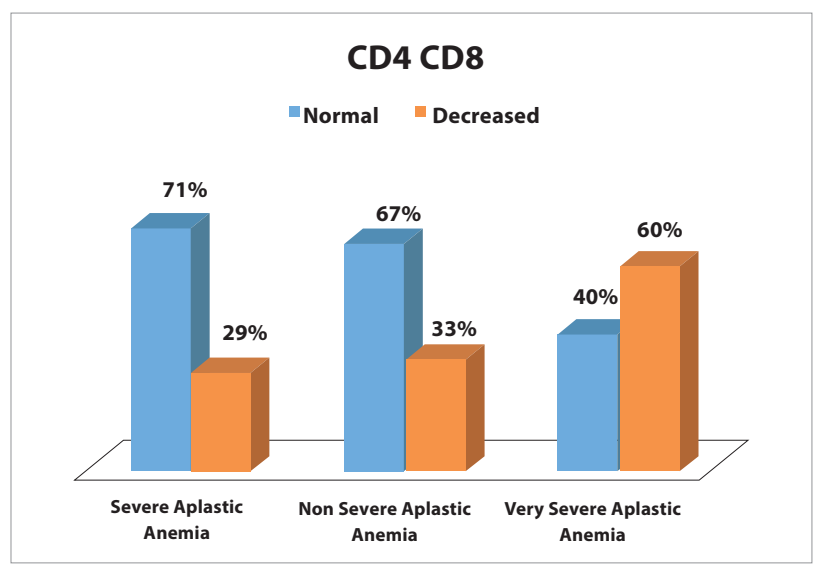

Fig. (2). CD4 and CD8 Cells in Aplastic Anemia. 


\section{DISCUSSION}

We found a very low Treg cell population in newly diagnosed acquired aplastic anaemia patients in our cohort. These results are similar to previously published findings by other groups that inappropriate immune mediate response plays a major role in the pathogenesis of Aplastic Anaemia; a mechanism somewhat similar to the other autoimmune disorders $[6,7$, 10]. The exact mechanism of $\mathrm{CD} 34+$ cell suppression is not known but there is substantial evidence of $\mathrm{CD} 8+\mathrm{T}$ cells expansion with release of inhibitory cytokines leading to destruction of hematopoietic stem cells [13]. The deficiency of Treg subset of CD4+ cells has been linked to many other autoimmune diseases like type I diabetes 1 [4], rheumatoid arthritis [15] and systemic lupus erythematosus [16]. Recent studies have implicated the role of a similar phenomenon in the pathogenesis of Aplastic Anaemia in West. Initially, the studies only documented the quantitative defect of Treg in AA [10], however, subsequently the qualitative defects were also documented [7]. The study provides some evidence to support the similar pathological phenomenon as a cause of Aplastic Anemia in Pakistani population in accordance with Western data. Meanwhile it has always been difficult to accurately identify the intrinsic defect of Treg in autoimmune disorders including AA.

\section{CONCLUSION}

In conclusion, the study provided some evidence that there is a quantitative defect of Treg in AA which causes immune dysregulation, ultimately responsible for the disease spectrum. Further studies are to be done to find out whether immunosuppressive therapy is able to correct the Treg deficiency in our patients with the normalization of peripheral blood counts along with haematopoietic recovery.

\section{CONFLICT OF INTEREST}

Declared none.

\section{ACKNOWLEDGEMENTS}

Declared none.

\section{REFERENCES}

[1] Young NS, Calado RT, Scheinberg P. Current concepts in the pathophysiology and treatment of aplastic anemia. Blood 2006; 108(8): 2509-19

DOI: 10.1182/blood-2006-03-010777

[2] Young NS, Bacigalupo A, March JC. Aplastic anemia: Pathophysiology and treatment. Bio Blood Marrow Transplant 2010; 16 (1 suppl): S119-S25.

DOI: $10.1016 /$ j.bbmt.2009.09.013

[3] Rosenfeld S, Follmann D, Nunez O, Young NS. Antithymocyte globulin and cyclosporine for severe aplastic anemia:
Association between hematologic response and long-term outcome. JAMA 2003; 289(9): 1130-5. DOI: 10.1001/jama.289.9.1130

[4] Yong NS. Pathophysiologic mechanism in acquired aplastic anemia. Hemat Am Soc Hematolo Educ Prog 2006; (1): $72-7$. DOI: 10.1182/asheducation-2006.1.72

[5] Perez-Diez A, Joncker NT, Choi K, et al. CD4 cells can be more efficient at tumor rejection than CD8 cells. Blood 2007; 109(12): 5346-54.

DOI: 10.1182/blood-2006-10-051318

[6] Kordasti S, Marsh J, Sufyan Al-Khans, et al. Functional characterization of CD4+ $\mathrm{T}$ cells in aplastic anemia. Blood 2012; 119 (9): 2033-43.

DOI: $10.1182 /$ blood-2011-08-368308

[7] Shi j, Ge M, Lu S, et al. Intrinsic impairment of CD4+CD25+regulatory $\mathrm{T}$ cells in acquired aplastic anemia. Blood 2012; 120 (8): 1624-32.

DOI: 10.1182/blood-2011-11-390708

[8] Buckner JH. Mechanism of impaired regulation by CD4+CD25+Foxp3+regulatory $\mathrm{T}$ cells in autoimmune disease. Nat Rev Immunol 2010; 10(12): 849-59.

DOI: $10.1038 /$ nri2889

[9] Kotsianidis I, Bouchliou I, Nakou E, et al. Kinetics function and bone marrow trafficking of CD4+ CD25+Foxp3+ regulatory $\mathrm{T}$ cells in myelodysplastic syndromes (MDS). Leukemia 2009; 23(3): 510-8.

DOI: $10.1038 /$ leu.2008.333

[10] Solomou EE, Rezvani k, Mielke S, et al. Deficient CD4+ CD25+Foxp3+ regulatory $\mathrm{T}$ cells in acquired anemia. Blood 2007; 110(5): 1603-16.

DOI: 10.1182/blood-2007-01-066258

[11] De Latour RP, Visconte V, Takaku T, et al. Th17 immune response contributes to the pathophysiology of aplastic anemia. Blood 2010; 116(20): 4175-184. DOI: 10.1182/blood-2010-01-266098

[12] Fillick SB, Bown N, Cavenagh J, et al. Guidelines for the diagnosis and management of adult aplastic anaemia. $\mathrm{Br} \mathrm{J}$ Haematol 2016; 172: 187-207.

DOI: $10.1111 /$ bjh. 13853

[13] Zang Y, Katsanis E. The complex pathophysiology of acquired aplastic anemia. Clin Exp Immunol 2015; 180: 361-70. DOI: 10.1111/cei.12605

[14] Long SA, Serosaletti K, Bollyky PL, et al. Defects in IL-2R signaling contribute to diminished maintenance of FOXP3 expression in $\mathrm{CD} 4+\mathrm{CD} 25+$ regulatory $\mathrm{T}$ cells of type I diabetic subjects. Diabetes 2010; 59(2): 407-15. DOI: 10.2337/db09-0694 
[15] Ehrenstein MR, Evans JG, Singh A, et al. Compromised functions of regulatory $\mathrm{T}$ cells in rheumatoid arthritis and reversal by anti-TNF alpha therapy. J Exp Med 2004; 2000(3): 277-85. DOI: $10.1084 / \mathrm{jem} .20040165$
[16] Fritsch RD, Shen X, Illei GG, et al. Abnormal differentiation of memory $\mathrm{T}$ cells in systemic lupus erythematosis. Arthritis Rheum 2006; 54(7): 2184-97. DOI: 10.1002/art.21943 